\title{
ASO Author Reflections: The Tumor Lysate, Particle-Loaded, Dendritic Cell Vaccine for Advanced-Stage Melanoma: Reflection on Personalized Cancer Vaccination
}

\author{
Alexandra M. Adams, $\mathrm{MD}^{1}$ (D), Timothy J. Vreeland, $\mathrm{MD}^{2,3}$, Guy T. Clifton, $\mathrm{MD}^{2,3}$, and \\ George E. Peoples, $\mathrm{MD}^{4}$ \\ ${ }^{1}$ Department of Surgery, Brooke Army Medical Center, San Antonio, TX; ${ }^{2}$ Department of Surgery, Uniformed Services \\ University of Health Sciences, Bethesda, MD; ${ }^{3}$ Department of Surgical Oncology, Brooke Army Medical Center, San \\ Antonio, TX; ${ }^{4}$ Cancer Vaccine Development Program, San Antonio, TX
}

\section{PAST}

Checkpoint inhibitor (CPI) and BRAF/MEK inhibitor therapies have provided new options for patients with advanced melanoma and have also been applied to the adjuvant setting. Unfortunately, not all patients respond to CPIs and their use is often limited by adverse events. Similarly, the responses to BRAF/MEK inhibitors are typically short-lived due to tumor evolution. ${ }^{1}$ Personalized cancer vaccination offers a unique approach to activate an individual's own immune system and to generate a tumorspecific response, including against unique neoantigens specific to the individual's tumor. However, clinical results with cancer vaccines in general, have been disappointing, with most trials conducted in the metastatic setting. ${ }^{2}$ Conversely, our study investigates the role of vaccination as adjuvant therapy for resected, advanced-stage melanoma.

\section{PRESENT}

Our randomized, double-blinded, placebo-controlled, phase IIb trial investigated tumor lysate, particle-loaded, dendritic cell (TLPLDC) vaccine administration to prevent recurrence in patients with resected stage III/IV melanoma

(C) Society of Surgical Oncology 2021

First Received: 11 February 2021

Accepted: 15 February 2021;

Published Online: 26 February 2021

A. M. Adams, MD

e-mail: lexy.adams16@gmail.com after completion of other standard-of-care (SoC) therapies. ${ }^{3}$ Co-primary endpoints were prespecified as intentionto-treat (ITT) 2-year disease-free survival (DFS) and pertreatment (PT) 2-year DFS, with the PT group defined as patients who completed the primary vaccine series (PVS). The PT group was included in the primary analysis as the active specific immunity generated by vaccination takes time to demonstrate its clinical effect, similar to trends observed with pembrolizumab, ${ }^{4}$ and patients with early recurrence did not receive the full treatment course. A total of 144 patients were enrolled, with 103 patients in the vaccine group and 41 in the control group. Notably, the SoC within this group of patients changed significantly during the conduct of our trial, and our protocol was amended to allow concurrent CPI therapy. The TLPLDC vaccine was demonstrated to be safe, with no difference in the rates of total adverse events between groups (35.9\% vaccine vs. $31.7 \%$ control; $p=0.632$ ). No statistical difference was found in the ITT 2-year DFS (38.5\% vs. $27.0 \% ; p=0.974$ ), but, according to $\mathrm{PT}$ analysis, 2-year DFS improved in the vaccinated patients $(62.9 \%$ vs. $34.8 \% ; p=0.041$ ).

\section{FUTURE}

Our analysis demonstrated that the TLPLDC vaccine is safe and well-tolerated and demonstrated statistically significant efficacy as adjuvant therapy for patients who completed the PVS. Meanwhile, indications for CPI have expanded into adjuvant treatment for stage III disease, but many patients do not respond to this therapy or cannot tolerate it. There is increasing evidence for a synergistic effect between CPIs and cancer vaccination, with a vaccine 
causing an initial T-cell infiltration into tumor, and CPI activating and protecting those $\mathrm{T}$ cells to lead to a more meaningful antitumor response. ${ }^{5}$ Our planned phase III trial will evaluate the efficacy of the TLPLDC vaccine plus CPI versus CPI alone in clinically disease-free patients with high-risk stage III/IV melanoma, and will better elucidate if this synergy translates to a clinical benefit.

DISCLOSURE George Peoples has received research funding to run this trial and works as a consultant to the company sponsoring the study. Alexandra M. Adams, Timothy J. Vreeland, and Guy T Clifton have no conflicts of interest to declare.

\section{REFERENCES}

1. Wahid M, Jawed A, Mandal RK, et al. Recent developments and obstacles in the treatment of melanoma with BRAF and MEK inhibitors. Crit Rev OncolHematol. 2018;125:84-8.
2. Clifton GT, Kohrt HE, Peoples GE. Critical issues in cancer vaccine trial design. Vaccine. 2015;33(51):7386-92.

3. Vreeland TJ, Clifton GT, Hale DF, et al. A Phase IIb randomized controlled trial of TLPLDC vaccine as adjuvant therapy after surgical resection of stage III/IV melanoma: a primary analysis. Ann Surg Oncol. 2021. https://doi.org/10.1245/s10434-021-097091.

4. Eggermont AMM, Blank CU, Mandala M, et al. Adjuvant pembrolizumab versus placebo in resected stage III melanoma. $N$ Engl J Med. 2018;378(19):1789-801.

5. Kleponis J, Skelton R, Zheng L. Fueling the engine and releasing the break: combinational therapy of cancer vaccines and immune checkpoint inhibitors. Cancer Biol Med. 2015;12(3):201-8.

Publisher's Note Springer Nature remains neutral with regard to jurisdictional claims in published maps and institutional affiliations. 